\title{
The diagnostic value of basophil activation tests in hypersensitivity reactions due to contrast media
}

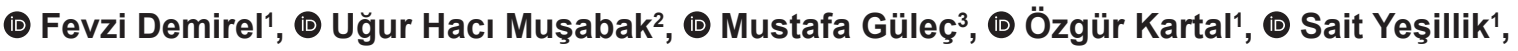 \\ (1) Abdullah Baysan ${ }^{4}$, (1) Aliye Aysel Pekel ${ }^{5}$, (1) Rahşan Ilıkçı Sağkan ${ }^{6}$, (1) Osman Şener ${ }^{1}$ \\ 1 University of Health Sciences Turkey, Gülhane Training and Research Hospital, Clinic of Immunology and Allergic Diseases, \\ Ankara, Turkey \\ 2Baskent University Hospital, Clinic of Immunology and Allergic Diseases, Ankara, Turkey \\ 3Private Guven Hospital, Clinic of Immunology and Allergic Diseases, Ankara, Turkey \\ 4 University of Health Sciences Turkey, Bozyaka Training and Research Hospital, Clinic of Immunology and Allergic Diseases, \\ Izmir, Turkey \\ 5Private Deren Laboratory, Ankara, Turkey \\ 6Usak University Faculty of Medicine, Department of Medical Biology, Usak, Turkey
}

\section{Date submitted:}

28.01.2021

Date accepted:

20.04.2021

Online publication date:

15.12.2021

\section{Corresponding Author:}

Fevzi Demirel, M.D., University of Health Sciences Turkey, Gülhane Training and Research Hospital, Clinic of Immunology and Allergic Diseases, Ankara, Turkey

drfevzidemirel@yahoo.com.tr

ORCID:

orcid.org/0000-0002-9711-6821

Keywords: Radiocontrast media, basophil activation test, CD63, CD203c

Presented in: This study was presented as a poster at the European Academy of Allergy and Clinical Immunology \& World Allergy organization Congress, June 22-26, 2013, Milan-Italy.

\begin{abstract}
Aims: Current tests used for the diagnosis of hypersensitivity reactions (HRs) due to radiocontrast media (RCM) are associated with important shortcomings. The present study investigated the diagnostic value of basophil activation test (BAT) in immediate HRs to RCM.

Methods: This was a cross-sectional study with prospective enrollment. The skin test (ST) with RCM was performed in cases with suspicious immediate RCM allergy. The patients were categorized into four groups (G); G1: positive ST to RCM; G2: negative ST to RCM; G3: no RCM reaction; and G4: controls with no history of RCM use. BAT was performed using flow cytometry to analyze CD63 and CD203c expression in basophils. Activated basophil percentage (ABP), stimulation index $(\mathrm{SI})$, mean fluorescence intensity (MFI), and the SI of the MFI (MFI SI) were compared across 4 groups.

Results: A total of 42 patients (female: $73.8 \%$, age 26 to 78 years) were evaluated. SI was greater in $\mathrm{G} 1(\mathrm{n}=7)$ than in $\mathrm{G} 3(\mathrm{n}=9)(\mathrm{p}=0.05)$; MFI was greater in $\mathrm{G} 1$ than in $\mathrm{G} 4(\mathrm{n}=17)(\mathrm{p}=0.03)$; and MFI SI was greater in $\mathrm{G} 1$ than in $\mathrm{G} 4(\mathrm{p}=0.03)$. Four subjects $(57.1 \%)$ in $\mathrm{G} 1$ had an ABP of $>5 \%$, 3 subjects $(42.8 \%)$ had a SI $\geq 2$, and 5 patients $(71.4 \%)$ had elevated $\mathrm{MFI}$; the corresponding figures in $\mathrm{G} 2(\mathrm{n}=9)$ were $2(22.2 \%), 1(11.1 \%)$ and $1(11.1 \%)$, respectively. All three criteria were positive only in 2 patients in $\mathrm{G} 1$.
\end{abstract}

Conclusions: This study suggests that, based on ABP, SI, and MFI assessments, BAT may represent a partially useful test in patients with a history of immediate RCM reactions. 


\section{Introduction}

Adverse reactions associated with radiocontrast media (RCM) generally occur within the first hour of administration and are termed as "immediate reactions". And delayed reactions are less frequent and occur $\geq 1$ hour after RCM administration $(1,2)$. Globally, each year more than 75 million imaging studies involving RCMs are performed and sometimes severe immunologic reactions may emerge $(3,4)$. Currently, no clinical sign or laboratory test that would allow us to predict individuals who are more likely to experience such hypersensitivity reactions (HR) exists $(5,6)$. The reported sensitivity of skin tests (ST) performed within 6 months of a hypersensitivity event for IgE-mediated immediate reactions ranges between $20 \%$ and $50 \%$, and these figures progressively decrease with increasing duration of time after the reaction (7). Due to their chemical characteristics, RCMs may trigger a reaction by direct stimulation of the effector cells (mast cells, eosinophils, basophils) responsible for a HR. Even in patients with a history of immediate RCM reaction, the ST may not be positive, or a negative ST cannot unequivocally rule out a HR, limiting the utility of these ST for screening purposes in daily clinical practice $(8,9)$.

Despite the identification of certain risk factors in previous large-scale studies (e.g. female gender, atopy, use of ionic $\mathrm{RCM}$ ), no test can definitively diagnose or exclude a RCM HR (10). On the other hand, establishing a confident diagnosis of RCM HR is very important from a clinical viewpoint. Canceling a significant imaging study due to possible HR based on an inadequately investigated RCM reaction may lead to untoward consequences in terms of diagnosis and treatment of a patient. Functional in vitro tests that can predict such reactions with higher sensitivity and specificity have been developed $(11,12)$. These tests are based on the measurement of mediators released from basophils. In this respect, the basophil activation test (BAT) is based on the detection of certain molecules produced by cell activation and presented to the cell surface. While basophils produce and release a wide range of mediators, they also express the activation markers on their surface. These alterations occurring at the cellular level are evaluated with the flow cytometry method. Major activation markers for basophils include CD63 and CD203c (13). BAT, which allows the detection of these molecules, has been shown to be valuable in the detection of reactions caused by a variety of substances including muscle relaxants, antibiotics, antiseptics, and plasma expanders (14-16). In previous studies, BAT has been used to confirm the results of ST or to assist in making clinical decisions among therapeutic options in patients. Therefore, the objective of this study was to evaluate both ST and BAT in patients presenting with immediate RCM HR history, to compare these two examinations, and to investigate the diagnostic value of BAT among patients in whom ST failed to diagnose RCM reactions.

\section{Methods}

\section{Study Groups}

This was a cross-sectional study with prospective enrollment. The participants were selected among the outpatients of the Immunology and Allergy Diseases Polyclinic of Gülhane Training and Research Hospital between October 2011 and October 2012. The main inclusion criterion was admission due to suspicious RCM allergy. The eligible patients with RCM allergy history were divided into two groups as those with an immediate or delayed type of reaction. The distinction between immediate and delayed RCM HR was made using the criteria proposed by Brockow et al. $(17,18)$. The severity of the reaction in immediate reactions was examined using the Ring and Messmer reaction severity scale (19). ST with RCMs were performed in the immediate reaction group.

Then, the patients were categorized into four study groups based on RCM use and ST positivity with RCM. Group (G) 1 consisted of the patients with immediate RCM reaction and positive ST to RCM; G2 consisted of the patients with immediate $\mathrm{RCM}$ reaction and negative ST to RCM; G3 consisted of healthy controls, in whom RCM was administered with no reaction; and G4 consisted of healthy volunteers without any history of RCM use. Then BAT was performed in all these four groups using the RCM, which was associated with the highest frequency of ST positivity.

The participants in both patient and control groups were at the age of 18 years or older and those who agreed to participate in this study were included in the study. Patients younger than 18 years of age despite having a history of immediate type reaction with RCM, patients with a history of late type RCM reaction, and patients with a history of allergic reaction to other drugs despite having a history of immediate type reaction with RCM were excluded from the study. It was paid attention that the patients in the control groups did not have a history of allergic reaction to other drugs. Written informed consent was obtained from all participants in the study groups. The study was approved by the Local Ethical Committee of Gülhane Training and Research Hospital, Ankara (date: 09.08.2011, protocol no: 177).

\section{Skin Test}

Skin prick test (SPT) and intradermal test using six different RCM were performed in patients with an immediate reaction history with RCM use $(17,18,20)$. In these tests the following RCMs were utilized: iobitridol (350 mg I/mL, Xenetix, Guebet, Villepinte, France), iodixanol (320 mg l/mL, Visipaque, Ireland), iomeprol (300 mg I/mL, lomeron, Italy), iohexol (300 mg I/mL, Omnipaque, GE Healthcare Ireland, Cork, Ireland), Iopromid (300 mg I/mL, Ultravist, Bayer Schering Pharma, Berlin, Germany), and sodium diatrizoate $(150 \mathrm{mg} \mathrm{I} / \mathrm{mL}$, Urografin, England). Histamine $(0.01 \%)$ and physiological saline were used as positive and negative controls. SPT performed with 
RCM at a dilution of 1/1 was administered on the volar surface of the forearm and the reading was performed 15 minutes after administration. SPT with $a \geq 3 \mathrm{~mm}$ induration as compared to negative control was considered positive $(18,21)$. If SPT was negative, 0.03-0.05 mL of RCM at a dilution of $1 / 100$ and 1/10, respectively, were administered via intradermal route on the volar surface of the forearm to form a bleb with a 3 to $5 \mathrm{~mm}$ diameter. The readings were performed 15 minutes after the injections. An induration of 3-5 mm, 6-10 mm, and 11-15 mm was graded $1+, 2+$, and $3+$, respectively $(18,22)$.

\section{Basophil Activation Test}

lobitridol (Xenetix, Guebet, Villepinte, France) at a concentration of $1 / 100$ was chosen for BAT testing, as this was the agent associated with the highest frequency of ST positivity and was used in most of the patients (23). Blood samples of 3-4 $\mathrm{mL}$ were obtained into K3 EDTA tubes from patients and healthy controls, and testing was performed within a maximum duration of 1 hour.

BAT was performed using Flow CAST ${ }^{\mathrm{R}}$ Highsens (Bühlmann Laboratories, BAT, Switzerland) flow cytometry kits containing lysing reactive with stimulation buffer containing calcium and heparin; stimulation control containing anti-FcERI monoclonal antibody (mAb); stimulation control containing $\mathrm{N}$-formylmethionyl-leucyl-phenylalanine (fMLP); and staining reactive consisting of anti-CD63-PE-DY647/anti-CD203c-PE-DY647/ anti-CCR3-PE mAb mixture. For BAT, 5 flow tubes were prepared, with the delivery of $50 \mu \mathrm{L}$ of peripheral blood in each. Fifty $\mu \mathrm{L}$ of stimulation buffer was added into PB (Patient Background tube); stimulation control containing $50 \mu \mathrm{L}$ of anti-FceRI mAB was added into PC 1 (patient control) tube; stimulation control containing $50 \mu \mathrm{L}$ of fMLP was added into PC 2 tube; $50 \mu \mathrm{L}$ of PBS was added into the basal tube, and finally, $50 \mu \mathrm{L}$ of RCM at a dilution of $1 / 100$ was added into the last tube. Also, $100 \mu \mathrm{L}$ of stimulation, the buffer was added into all tubes. The antibody mixture containing anti-CD63-PE-DY647/anti-CD203c-PEDY647/anti-CCR3-PE was dispensed into each tube at a volume of $20 \mu \mathrm{L}$. The tubes were gently mixed and were incubated for 15 minutes in a water bath for $37^{\circ} \mathrm{C}$ with closed caps. The tubes were kept at room temperature in a dark space for erythrocyte lysis. The tubes prepared in this manner were analyzed within 1 hour using a FACS Canto flow cytometry device (BD, San Jose, USA) and FACS Diva software. For analyses, a total of 200.000 cells were counted and the entry of 300 cells into the basophilgate was targeted. When the CD63+ CD203c expression was greater than $5 \%$ and the stimulation index (SI) was $\geq 2$, the result was considered positive (Figure 1,2). SI was defined as the ratio of CD63+ CD203c the expression after in vitro stimulation with RCM to basal CD63+ CD203c expression (23).

\section{Statistical Analysis}

For all statistical analyses, Statistical Package for the Social Sciences (SPSS) statistical software package (SPSS 15.0, SPSS Inc., Chicago, IL, USA) was used. Descriptive statistics were expressed as mean \pm standard deviation and percentage. Normality of the tests was evaluated with Kolmogorov-Smirnov test. For multiple group comparisons, one-way ANOVA with Bonferroni correction was utilized. Qualitative data were compared with chi-square analysis. $P$ value of less than 0.05 was considered statistically significant. The sensitivity of the ST was defined as the ratio of patients with positive ST for RCM to the patients with RCM reaction history. BAT sensitivity was defined as the number of true positive cases/number of true

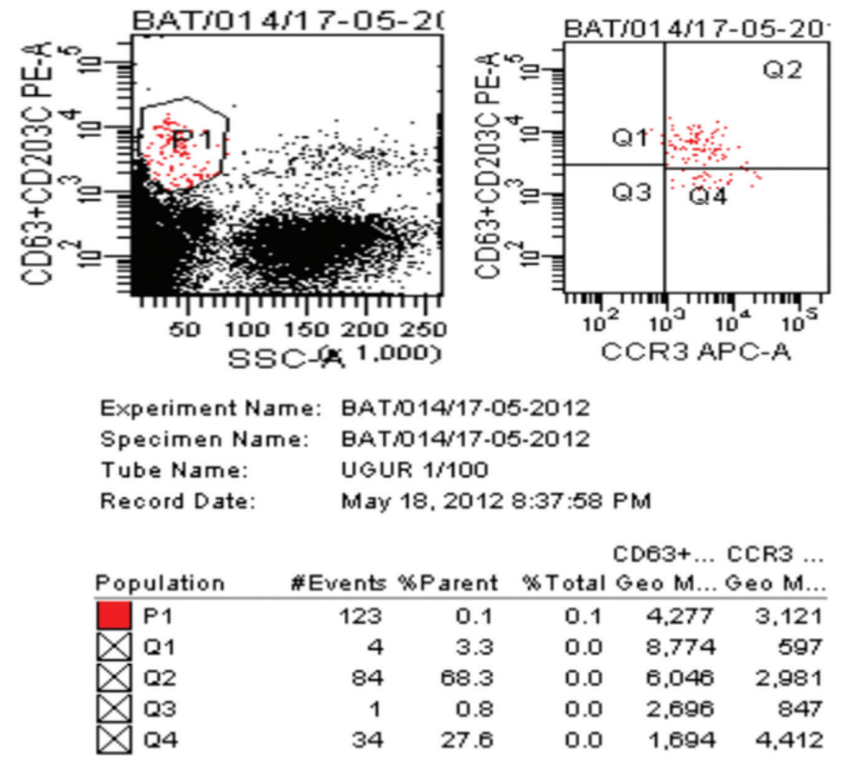

Figure 1. Positive basophil activation test after in vitro stimulation with $1 / 100$ radiocontrast media

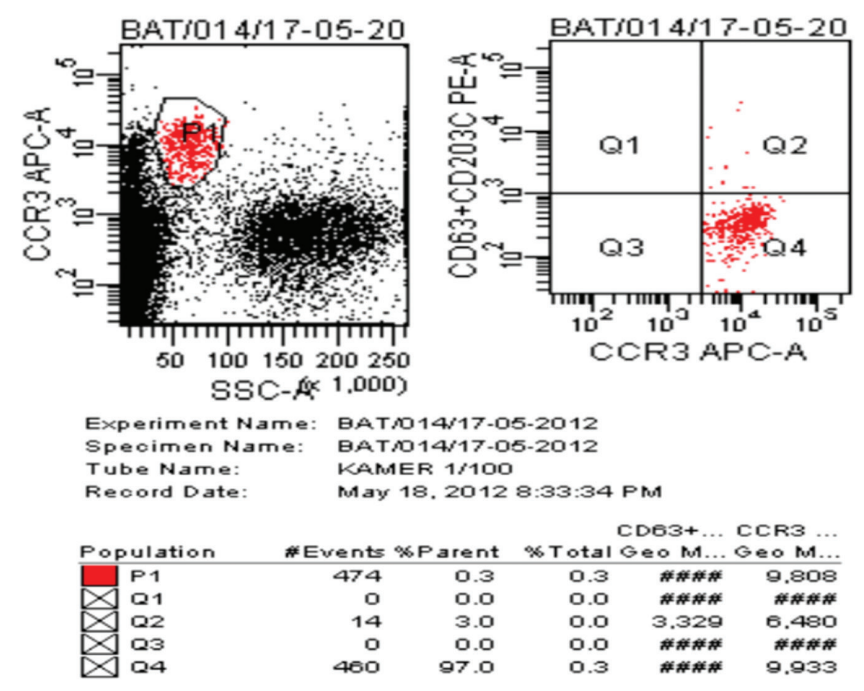

Figure 2. Negative basophil activation test after in vitro stimulation with $1 / 100$ radiocontrast media 
positive cases+false-negative cases, while BAT specificity was defined as the number of true negative cases/number of true negative cases+false-positive cases. The positive predictive value (PPV) for BAT was formulated as the number of true positive cases/number of true positive cases+false-positive cases, and the negative predictive value (NPV) for BAT was formulated as the number of true negative cases/number of true negative cases+false-negative cases.

\section{Results}

A total of 100 patients who were admitted to the immunology and allergy disease outpatient with suspicion of RCM allergy were included. There were 88 patients $(88 \%)$ with immediate and 12 patients $(12 \%)$ with delayed reactions. Patients with immediate reaction history underwent ST with six different RCMs. ST showed iobitridol positivity in 11 of 77 patients, iodixanol positivity in 1 of 36 patients, iomeprol positivity in 4 of 75 patients, iohexol positivity in 2 of 42 patients, lopromid positivity in 1 of 5 patients, and sodium diatrizoate positivity in 1 of 9 patients (Table 1 ).

A total of 42 subjects [ 12 male $(28.6 \%), 30$ female $(71.4 \%)$ ] aged between 26 and 78 years formed four study groups.
Group 1 consisted of 7 patients, G2 consisted of 9 patients, G3 consisted of 9 healthy controls, and G4 consisted of 17 healthy volunteers. All the participants in these groups underwent BAT using the RCM containing iobitridol. The four study groups were compared with respect to demographic characteristics. No significant differences between the groups were observed in terms of the parameters such as gender, presence of gadolinium allergy, family history of allergic disorders, recall of suspectedculprit RCM, reaction severity, and procedure during which the reaction had occurred. There were statistically significant differences between the groups related to age, and admission to the clinic but these differences did not affect the test results (Table 2). ST were performed 3 to 120 months after the RCM reaction. When subjects with a positive reaction history were compared regarding the time elapsed between reaction and RCM ST, no significant differences were detected. Patients in G1 and G2 did not exhibit significant differences with respect to symptoms occurring during the reaction.

When BAT results after in vitro stimulation with RCM were examined in the overall study groups, an increase in the percentage of activated basophils was found in 4 patients $(57.1 \%)$ in $\mathrm{G} 1,7$ patients in $\mathrm{G} 2(77.7 \%), 2$ patients $(22.2 \%)$ in $\mathrm{G} 3$, and

Table 1. Skin test results of patients with immediate type reaction history with radio contrast media $(n=88)$

\begin{tabular}{|c|c|c|c|c|c|c|}
\hline $\mathrm{RCM}^{*}$ & $\begin{array}{l}\text { lobitridol } \\
(n=77)\end{array}$ & $\begin{array}{l}\text { Iodixanol } \\
(n=36)\end{array}$ & $\begin{array}{l}\text { lomeprol } \\
(n=75)\end{array}$ & $\begin{array}{l}\text { Iohexol } \\
(n=42)\end{array}$ & $\begin{array}{l}\text { lopromid } \\
(n=5)\end{array}$ & $\begin{array}{l}\text { Sodium diatrizoate } \\
(n=9)\end{array}$ \\
\hline Positive SPT & 0 & 0 & 1 & 1 & 0 & 0 \\
\hline Positive $1 / 10$ IDT & 5 & 0 & 1 & 0 & 1 & 1 \\
\hline Positive $1 / 100$ IDT & 6 & 1 & 2 & 1 & 0 & 0 \\
\hline Total positive test results, $\mathrm{n}(\%)$ & $11(14.2)$ & $1(2.7)$ & $4(5.3)$ & $2(4.7)$ & $1(20)$ & $1(11.1)$ \\
\hline Total negative test results, $\mathrm{n}(\%)$ & $66(85.8)$ & $35(97.3)$ & $71(94.7)$ & $40(95.3)$ & $4(80)$ & $8(88.9)$ \\
\hline
\end{tabular}

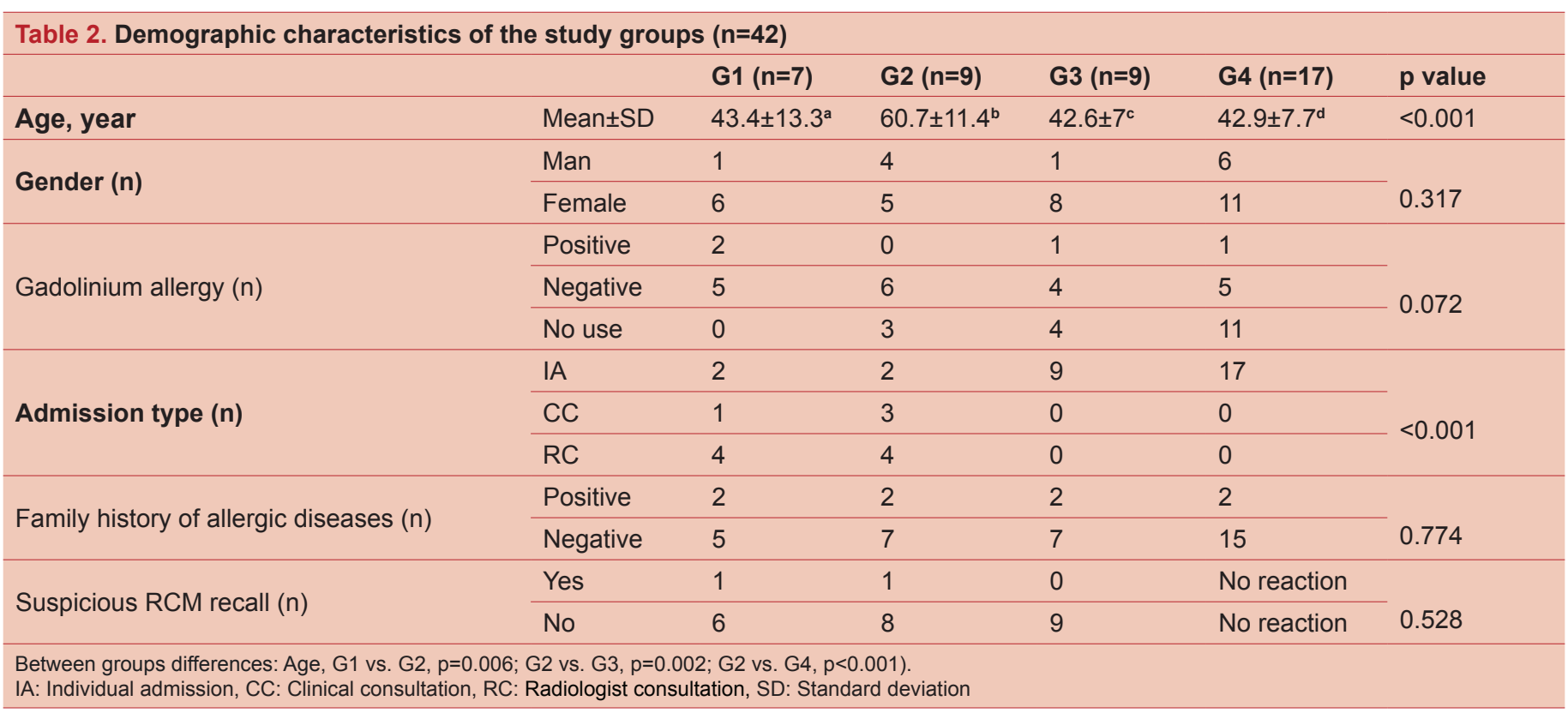


9 patients $(52.9 \%)$ in G4. The sensitivity comparison between G1 and G2 based on positive BAT results showed that the sensitivity was $57.1 \%$, specificity was $22.2 \%$, PPV was $36.3 \%$, and NPV was $40 \%$. When the ST results were disregarded and patients with positive reaction history ( $G 1$ and $G 2$ ) and negative reaction history ( $\mathrm{G} 3$ and $\mathrm{G} 4$ ) were taken into consideration, the sensitivity was $68.7 \%$, specificity was $57.6 \%$, PPV was $50 \%$, and NPV was $75 \%$. BAT results after in vitro stimulation with $\mathrm{RCM}$ and basal BAT results were examined in the overall study groups, the number of patients with a basophil percentage $>5 \%$ was $3(42.8 \%)$ in $\mathrm{G} 1,2(22.2 \%)$ in $\mathrm{G} 2,1$ (11.1\%) in G3, and 5 $(29.4 \%)$ in G4. When G1 and G2 were considered with respect to this positive BAT result, the sensitivity, specificity, PPV, and NPV were $42.8 \%, 77.7 \%, 60 \%$, and $63.6 \%$, respectively. When the ST was not taken into consideration and the groups with (G1 and G2) and without (G3 and G4) apositive history of reaction were examined, the rates of sensitivity, specificity, PPV, and NPV were $31.2 \%, 76.9 \%, 45.4 \%$, and $64.5 \%$, respectively. Overall, a SI value of $\geq 2$ was observed in 3 (42.8\%), 1 (11.1\%), $1(11.1 \%)$, and $2(11.7 \%)$ of the patients in G1, G2, G3, and $\mathrm{G} 4$, respectively. When $\mathrm{SI} \geq 2$ patients were considered to have positive BAT result in G1 and G2, the sensitivity, specificity, PPV, and NPV were $42.8 \%, 88.8 \%, 75 \%$, and $66.6 \%$, respectively. When the ST was excluded, the assessment among groups with a history of reaction ( $\mathrm{G} 1$ and $\mathrm{G} 2$ ) and without history of reaction (G3 and G4) showed a sensitivity, specificity, PPV, and NPV of $25 \%, 88.4 \%, 57.1 \%$, and $65.7 \%$, respectively.

In the overall study groups, the mean fluorescence intensity (MFI) increased in 5 (71.4\%), 1 (11.1\%), 4 (44.4\%), and 8 $(47.05 \%)$ of the patients in G1, G2, G3, and G4, respectively, after in vitro stimulation with RCM. When this increase was considered as a positive BAT result in G1 and G2, the sensitivity, specificity, PPV, and NPV were found to be $71.4 \%, 88.8 \%$, $83.3 \%$, and $80 \%$. When the skin test results were excluded and groups with (G1, G2) or without (G3, G4) a history of reaction were considered, the sensitivity, specificity, PPV, and NPV were $37.5 \%, 53.8 \%, 33.3 \%$, and $58.3 \%$, respectively. In all groups, an MFI SI $\geq 2$ was determined in 2 patients $(28.5 \%)$ in $\mathrm{G} 1,1$ patient (11.1\%) in G2, 2 patients (22.2\%) in G3, and 2 patients $(11.7 \%)$ in G4. When MFI SI values indicating a positive BAT result were taken into consideration in $\mathrm{G} 1$ and $\mathrm{G} 2$, the sensitivity, specificity, PPV, and NPV were $28.5 \%, 88.8 \%, 66.6 \%$, and $61.5 \%$, respectively. When the ST results were excluded, an assessment of groups with (G1 and G2) or without (G3 and G4) a history of reaction showed a sensitivity, specificity, PPV, and NPV of $18.75 \%, 84.6 \%, 42.8 \%$, and $62.8 \%$, respectively.

The four study groups were also compared regarding the following main laboratory results: white blood cell (WBC), eosinophil percentage, eosinophil count, basophil percentage, basophil count, total immunoglobulin E, basal BAT, BAT after $\mathrm{RCM}, \mathrm{SI}$, basal MFI, MFI after RCM, and MFI SI. There were significant differences between $\mathrm{G} 1$ and $\mathrm{G} 3$ in terms of WBC count $(p=0.046), G 2$ and $G 3$ in terms of eosinophil count $(p=0.025), G 1$ and $G 3$ in terms of $S I(p=0.05), G 1$ and G4 in terms of MFI at 1/100 RCM ( $p=0.03)$, and $\mathrm{G} 1$ and $\mathrm{G} 4$ in terms of MFI SI $(p=0.03)$ (Table 3).

\section{Discussion}

Although an IgE-mediated process has been implicated, the presence of specific IgE against RCM could only rarely be identified in ST (10). In this study, the potential role of BAT in diagnosing immediate HR due to RCM was investigated. The ST with several RCMs yielded a positive result in $13(14.7 \%)$ patients and a negative result in $75(85.3 \%)$ patients. According

\begin{tabular}{|c|c|c|c|c|c|c|}
\hline & & G1 (n=7) & $\mathbf{G} 2(n=9)$ & G3 (n=9) & G4 (n=17) & $p$ value \\
\hline 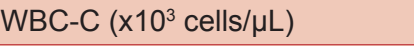 & Mean $\pm S D$ & $6700 \pm 787$ & $6544 \pm 1005$ & $4911 \pm 1395$ & $5682 \pm 1434$ & 0.019 \\
\hline Eos-C. ( $\left(x 10^{3}\right.$ cells $\left./ \mu \mathrm{L}\right)$ & Mean $\pm S D$ & $0.158 \pm 0.04$ & $0.184 \pm 0.08$ & $0.077 \pm 0.03$ & $0.144 \pm 0.08$ & 0.029 \\
\hline Per. of Bas. & $\%$ & 0.9 & 0.8 & 0.8 & 0.9 & 0.739 \\
\hline Percentage of BAT basal & $\%$ & 4.5 & 3.01 & 6.3 & 5.5 & 0.415 \\
\hline Percentage of BAT 1/100 RCM & $\%$ & 7.2 & 3.5 & 3.4 & 3.9 & 0.395 \\
\hline $\mathrm{SI}$ & Mean \pm SD & $2.1 \pm 2.2$ & $1.3 \pm 0.5$ & $0.6 \pm 0.4$ & $0.9 \pm 0.5$ & 0.043 \\
\hline MFI basal & Mean $\pm S D$ & $2977 \pm 1906$ & $2981 \pm 1316$ & $4061 \pm 2489$ & $4403 \pm 3014$ & 0.511 \\
\hline
\end{tabular}


to these data, the sensitivity observed in our study is lower than the previously reported figure of $20 \%(7,10,21,22,24)$. In another study, 270 patients with a history of reaction to RCM also underwent skin testing, and the predictive value and sensitivity of ST for ionic RCMs were reported to be $1.2 \%$ and $3.7 \%$, respectively (25). When this latter piece of evidence is taken into consideration, the observed sensitivity of ST in our study was higher. BAT has also been introduced as an alternative or complementary method to skin testing in the assessment of immediate HRs due to RCM (26). Although some case reports have suggested that BAT may have a significant role in the identification of early $\mathrm{RCM}$ reactions, published clinical data regarding its predictive value is very scarce $(15,23,27)$.

On the other hand, the presence of a patient group with contrast media use but no reaction history (i.e., G3) may be considered as a distinguishing aspect of our study. Patient and control groups described above facilitate the assessment of BAT results in the context of the study design. However, one disadvantage of our study was the inclusion of a smaller patient population in $\mathrm{G} 1$, as compared to previous similar studies $(7<11)$ (23). In general, the stimulant doses used in BAT have been partially established thanks to previous investigations. However, BAT testing for RCM has been relatively limited. In one previous study, $1 / 10$ and $1 / 100$ dilutions of 5 separate RCM were used irrespective of the results of the ST, and in vitro stimulation with 1/100 RCM was found to be associated with high sensitivity and specificity for BAT, correlating well with clinical manifestations (23). In the current study, since the culprit RCM could not be generally recalled, 1/100 concentration of the RCM associated with the highest frequency of positive results in ST was used as a stimulant for BAT.

The basophilic activation marker utilized in BAT has crucial importance with regard to test results (28). CD63 was generally used as the basophilic activation marker in previous BAT applications used for the diagnostic examination of drug HRs (29). However, the use of CD63 alone is associated with certain shortcomings. For instance, CD63 can be hardly identified in resting cells and in addition to basophils, may also be expressed by some other cell types, such as platelets. Also, it may take up to 15-25 minutes before the expression reaches the maximum level. Challenges associated with CD63 expression may also lead to false-negative results. On the other hand, CD203c is the only marker that is not expressed by other peripheral blood cells and that is expressed by basophils both in the resting and active state. Expression reaches the maximum more rapidly, i.e., within 5 to 10 minutes, as compared to CD63. On the other hand, CD203c expression in basophils, which is not as strong as that of CD63, may be affected by many substances other than the real stimulus, and thus may easily be associated with false-positive results. For these reasons, a BAT kit containing both CD63 and CD203c activation markers has been utilized in the current study to improve the reliability and accuracy of our results. To the best of our knowledge, this study is the first of its kind in using both CD63 and CD203c as basophilic activation markers for a BAT procedure aiming at detecting RCM hypersensitivity.

Basal active basophil percentage (ABP) after in vitro stimulation with $\mathrm{RCM}, \mathrm{SI}$; basal $\mathrm{MFI}, \mathrm{MFI}$ after in vitro stimulation with RCM, and MFI SI values were obtained with BAT. Also, several other laboratory parameters that could assist in interpreting $\mathrm{HR}$, including the WBC, eosinophil count, eosinophil percentage, basophil count, basophil percentage, and total immunoglobulin $\mathrm{E}$, were examined. Comparisons between the groups showed higher WBC count in $\mathrm{G} 1$ than in $\mathrm{G} 3$, higher eosinophil count in $\mathrm{G} 2$ than in $\mathrm{G} 3$, higher $\mathrm{SI}$ in $\mathrm{G} 1$ than in $\mathrm{G} 3$, higher MFI after RCM in G1 than in G4, and higher MFI SI in G1 than in G4. The higher parameters were detected in $\mathrm{G} 1$ and $\mathrm{G} 2$ groups. A higher number of parameters that were significantly elevated (e.g., WBC count, eosinophil count) suggests that the HR may persist in these individuals. On the other hand, SI BAT, $\mathrm{MFI}$, and MFI SI after in vitro stimulation with RCM, which are responsible for the difference between the groups of G1 and G3 and $\mathrm{G} 4$, have a major significance in terms of confirming the RCM reaction history with BAT. In other studies that utilized BAT, the criteria for positivity were generally not fully standardized. In one study utilizing RCMs for stimulation, expression of CD63+ basophils $>5 \%$ and a $\mathrm{SI} \geq 2$ was considered as a positive result (23). In our study, the test results were interpreted using the same criteria, although additional factors that might have an impact in terms of the outcome were evaluated, including an increase in BAT expression after in vitro stimulation with RCM (no upper limit defined), increase in MFI, and an MFI SI of $\geq 2$. The sensitivity, specificity, and predictive value of the BAT were first compared between the groups with a history of reaction on the basis of the ST (i.e., G1, and G2), and then between all groups regardless of the history of a reaction.

A high level of sensitivity, specificity, PPV, and NPV values was achieved when parameters such as an $\mathrm{ABP}>5 \%$ and $\mathrm{SI} \geq 2$ were considered as the criteria for positivity in the assessments in G1 and G2. However, in contrast with some previous studies, the highest degree of sensitivity, specificity, PPV and NPV were obtained when the increase in MFI compared to baseline after stimulation with RCM was considered (sensitivity $71.4 \%$, specificity $88.8 \%$, PPV $83.3 \%$, and NPV $80 \%$ ). These results are in line with previously reported numbers such as a sensitivity of $50 \%$ and a specificity of $90.7 \%$ (23). Regarding the reliability of the tests of our study, the highest specificity and NPV were obtained when the criteria consisting of elevated MFI and $\mathrm{SI} \geq 2$ were utilized. Also, when the overall study groups were compared, SI, MFI, and MFI SI values were significantly higher in the patient groups (Table 1). When the results of this comparison and the resultant sensitivity, specificity, PPV, and 
NPV were collectively considered, it may be assumed that a SI $\geq 2$ may be considered to show a positive result if BAT testing is performed in subjects with suspected RCM reaction. When an assessment among patients in $\mathrm{G} 1$ and $\mathrm{G} 2$ was made regarding $\mathrm{ABP} \geq 5 \%$, a $\mathrm{SI} \geq 2$, and $\mathrm{MFI}$ elevation as compared to baseline after stimulation with RCM, it was found that 4 patients $(57.1 \%)$ had $A B P \geq 5 \%, 3$ patients $(42.8 \%)$ had $\mathrm{SI} \geq 2$, and 5 patients $(71.4 \%)$ had elevated MFI in G1. All three positivity criteria were present in only 2 patients $(28.5 \%)$ in G1. On the other hand, 2 patients (22.2\%) had an ABP >5\%, 1 patient (11.1\%) had $\mathrm{SI} \geq 2$, and 1 patient (11.1\%) had elevated MFI in G2. All three positivity criteria were not present in G2.

Based on the percentage of activated basophils, SI, and MFI results, we may suggest that BAT alone does not seem to be as useful as skin tests in patients with a history of immediate RCM reaction history. When a good level of specificity for BAT is considered between $88.4 \%$ and $100 \%$ based on the use of all allergens, the elevations in SI and MFI in our study may partly provide this specificity. However, our results also showed that BAT could confirm only a fraction of the patients with immediate RCM reactions, in whom ST was positive, while it did not seem to provide meaningful contributions in those with negative ST reaction to $\mathrm{RCM}$. When patients with a history of reaction were considered, an ABP $>5 \%$, SI $\geq 2$, and $\mathrm{MFI}$ elevation after RCM stimulation provided higher sensitivity, specificity, PPD, and NPD as compared to other parameters, and therefore, their combined use may be important for the reliability of the test. The failure to achieve BAT positivity using these parameters in some patients may be accounted for by the prolonged duration between the reaction and testing time, or by the fact that certain reactions may not be mediated by the degranulation of basophils. While this is the second study systematically analyzing the accuracy of BAT in immediate RCM reactions, it is the first to utilize two different basophilic activation markers for RCM reactions to obtain complementary and more accurate results. When the findings of our study are taken into consideration, we may suggest that our study was partially successful in showing a consistency between ST results and BAT, and that BAT may potentially be used as a diagnostic tool, and particularly a confirmatory test in some cases of RCM related immediate HRs. It appears from these results that combined use of both ST and BAT may be more useful in the diagnosis of RCM related immediate HRs. Combined use of ST/BAT may represent an alternative option for the identification of a safe RCM in patients with a previous history of RCM and also for screening RCM related immediate HRs prior to RCM administration in high-risk patients. In the light of the currently published data, it appears that two separate activation markers have never been measured simultaneously in studies examining the diagnostic accuracy of BAT in RCM reactions. This situation gives a privilege to our study.
On the other hand, this study has some limitations. One of them is the low number of patients in the groups. The other important limitation of the study is that BAT test was performed only with one RCM (iobitridol). Moreover, since it was not ethically possible to carry out skin testing with RCMs in control subjects, the specificity and predictive value of our skin testing could not be estimated.

\section{Conclusion}

BAT represents a novel and promising diagnostic tool for immediate-type drug HRs involving IgE-mediated mast cell and basophil activation. Although BAT was successful only in a certain proportion of our patients with a history of RCM, the results of the current study seem to be supportive of this hypothesis. BAT may also provide a safe alternative testing tool in cases where routine in vitro diagnostic methods do not allow alternative testing, or in situations where clinical suspicions need to be confirmed (despite negative test results) in patients for whom ST or drug provocation tests are potentially dangerous, particularly if several drugs need to be tested altogether. However, since BAT is mostly an investigational method, further clinical studies are warranted to extrapolate its use into daily clinical practice. On the other hand, since BAT studies on RCM are relatively few and mostly involve case reports, studies with a larger sample size are required to obtain more accurate sensitivity, specificity, PPV, and NPV data and to confirm these observations.

\section{Ethics}

Ethics Committee Approval: The study was approved by the Local Ethical Committee of Gülhane Training and Research Hospital, Ankara (date: 09.08.2011, protocol no: 177).

Informed Consent: Written informed consent was obtained from all participants in the study groups.

Peer-review: Externally peer-reviewed.

\section{Authorship Contributions}

Concept: F.D., Ö.K., A.B., O.Ş., Design: F.D., U.H.M., M.G., Ö.K., S.Y., Data Collection or Processing: F.D., S,Y., A.B., Analysis or Interpretation: U.H.M., M.G., A.A.P., R.I.S., Literature Search: F.D., Writing: F.D.

Conflict of Interest: No conflict of interest was declared by the authors.

Financial Disclosure: The authors declared that this study received financial support from Gülhane Training and Research Hospital Scientific Research Board, Ankara (AR-2011/27).

\section{References}

1. Gelincik A. Radyokontrast Maddelere Karşı Gelişen Reaksiyonlar. Türkiye Klinikleri J Int Med Sci. 2006;2:2833. 
2. Christiansen C. Hypersensitivity reactions to iodinated contrast media: an update, In: Pichler W, editor. Drug hypersensitivity, 1st ed. Basel: Karger. 2007. p. 140-148.

3. Brockow K. Immediate and delayed reactions to radiocontrast media: is there an allergic mechanism? Immunol Allergy Clin North Am. 2009;29:453-468.

4. Brockow K, Ring J. Anaphylaxis to radiographic contrast media. Curr Opin Allergy Clin Immunol. 2011;11:326-331.

5. Caro JJ, Trindade E, McGregor M. The risks of death and of severe nonfatal reactions with high- vs low-osmolality contrast media: a meta-analysis. AJR Am J Roentgenol. 1991;156:825-832.

6. Guéant-Rodriguez RM, Romano A, Barbaud A, Brockow K, Guéant JL. Hypersensitivity reactions to iodinated contrast media. Curr Pharm Des. 2006;12:3359-3372.

7. Brockow K, Romano A, Aberer W, et al. Skin testing in patients with hypersensitivity reactions to iodinated contrast media - a European multicenter study. Allergy. 2009;64:234-241.

8. Laroche D, Namour F, Lefrançois C, et al. Anaphylactoid and anaphylactic reactions to iodinated contrast material. Allergy. 1999;54 Suppl 58:13-16.

9. Clement O, Dewachter P, Mouton-Faivre C, et al. Immediate Hypersensitivity to Contrast Agents: The French 5-year CIRTACI Study. EClinicalMedicine. 2018;1:51-61.

10. Goksel O, Aydın O, Atasoy C, et al. Hypersensitivity reactions to contrast media: prevalence, risk factors and the role of skin tests in diagnosis--a cross-sectional survey. Int Arch Allergy Immunol. 2011;155:297-305.

11. Romano A, Demoly P. Recent advances in the diagnosis of drug allergy. Curr Opin Allergy Clin Immunol. 2007;7:299303.

12. Ebo DG, Hagendorens MM, Bridts $\mathrm{CH}$, De Clerck LS, Stevens WJ. The basophil activation test in immediate drug allergy. Acta Clin Belg. 2009;64:129-135.

13. Ebo DG, Bridts CH, Hagendorens MM, Aerts NE, De Clerck LS, Stevens WJ. Basophil activation test by flow cytometry: present and future applications in allergology. Cytometry B Clin Cytom. 2008;74:201-210.

14. Hausmann OV, Gentinetta T, Bridts CH, Ebo DG. The basophil activation test in immediate-type drug allergy. Immunol Allergy Clin North Am. 2009;29:555-566.

15. Abuaf $\mathrm{N}$, Rostane $\mathrm{H}$, Rajoely $\mathrm{B}$, et al. Comparison of two basophil activation markers CD63 and CD203c in the diagnosis of amoxicillin allergy. Clin Exp Allergy. 2008;38:921-928.
16. Rodríguez-Trabado A, Cámara-Hijón C, Ramos-Cantariño A, et al. Basophil activation test for the in vitro diagnosis of nonsteroidal anti-inflammatory drug hypersensitivity. Allergy Asthma Proc. 2008;29:241-249.

17. Kopp AF, Mortele KJ, Cho YD, Palkowitsch P, Bettmann MA, Claussen CD. Prevalence of acute reactions to iopromide: postmarketing surveillance study of 74,717 patients. Acta Radiol. 2008;49:902-911.

18. Brockow K, Christiansen C, Kanny G, et al. Management of hypersensitivity reactions to iodinated contrast media. Allergy. 2005;60:150-158.

19. Ring J, Messmer K. Incidence and severity of anaphylactoid reactions to colloid volume substitutes. Lancet. 1977;1:466469.

20. Barbaud A, Gonçalo M, Bruynzeel D, Bircher A; European Society of Contact Dermatitis. Guidelines for performing skin tests with drugs in the investigation of cutaneous adverse drug reactions. Contact Dermatitis. 2001;45:321328.

21. Kvedariene V, Martins P, Rouanet L, Demoly P. Diagnosis of iodinated contrast media hypersensitivity: results of a 6-year period. Clin Exp Allergy. 2006;36:1072-1077.

22. Trcka J, Schmidt C, Seitz CS, Bröcker EB, Gross GE, Trautmann A. Anaphylaxis to iodinated contrast material: nonallergic hypersensitivity or IgE-mediated allergy? AJR Am J Roentgenol. 2008;190:666-670.

23. Ebo DG, Schuerwegh A, Stevens WJ. Anaphylaxis to starch. Allergy. 2000;55:1098-1099.

24. Dewachter $P$, Laroche D, Mouton-Faivre $C$, et al. Immediate reactions following iodinated contrast media injection: a study of 38 cases. Eur J Radiol. 2011;77:495-501.

25. Yamaguchi K, Katayama H, Kozuka T, Takashima T, Matsuura K. Pretesting as a predictor of severe adverse reactions to contrast media. Invest Radiol. 1990 Sep;25 Suppl 1:S22-3.

26. Romano A, Demoly P. Recent advances in the diagnosis of drug allergy. Curr Opin Allergy Clin Immunol. 2007;7:299303.

27. Dewachter P, Nicaise-Roland P, Kalaboka S, Lefèvre J, Chollet-Martin S. Anaphylaxis to amidotrizoate proved by skin testing and flow cytometry-based basophil activation test. Allergy. 2009;64:501-502.

28. Chirumbolo S. Basophil activation test in allergy: time for an update? Int Arch Allergy Immunol. 2012;158:99-114.

29. Sanz ML, Gamboa PM, Mayorga C. Basophil activation tests in the evaluation of immediate drug hypersensitivity. Curr Opin Allergy Clin Immunol. 2009;9:298-304. 